\title{
Effects of host modulation by nonsteroidal anti-inflammatory drugs on periodontal disease: a systematic review and meta-analysis
}

\author{
Dae-Young Kang, In-Woo Cho', Hyun-Seung Shin ${ }^{1}$, Hyeong-Sik Ahn², Hyun-Jung Kim²*, Jung-Chul Park ${ }^{1 *}$ \\ 'Department of Periodontology, College of Dentistry, Dankook University, Cheonan, Republic of Korea \\ ${ }^{2}$ Institute for Evidence-based Medicine, Department of Preventive Medicine, College of Medicine, Korea University, Seoul, \\ Republic of Korea
}

Purpose: Nonsteroidal anti-inflammatory drugs that prohibit biosynthesis of arachidonic acid metabolites have been considered potent host modulation agents. The aim of this review was to determine the effect of nonsteroidal anti-inflammatory drugs adjunctive with nonsurgical periodontal treatment in patients with periodontal disease. Materials and Methods: Three electronic databases were searched to identify relevant studies. The methodological quality and mean differences of the change in clinical attachment level and probing depth were analyzed according to Cochrane review methods. Results: Twelve studies were included in the methodological assessment and nine studies were suitable for inclusion in the meta-analysis. The mean difference in the clinical attachment level gain did not differ significantly between the nonsteroidal anti-inflammatory drugs and control groups at any observation time. The highest mean difference in clinical attachment level gain was $0.30 \mathrm{~mm}$ at 4 weeks $(95 \%$ confidence interval $=-0.37$ to 0.97$)$. There was a significant mean difference in the probing depth reduction, of $0.34 \mathrm{~mm}(95 \%$ confidence interval $=$ 0.29 to 0.40 ) at 6 weeks. Conclusion: Therefore, nonsteroidal anti-inflammatory drugs have additional therapeutic effect when administrated with nonsurgical periodontal treatment. (J Dent Rehabil Appl Sci 2017;33(1):7-18)

Key words: anti-inflammatory agents, non-steroidal; drug therapy; meta-analysis; periodontal diseases; prostaglandins

\section{Introduction}

Periodontitis is an infectious disease initiated by periodontal pathogens. ${ }^{1}$ It is well known that periodontal disease primarily develops due to bacterial infection. However, the initiation and progression of the diseases can vary among individuals based on genetic traits, systemic conditions, and environmental factors. ${ }^{2-4}$ There is a large body of literature showing that both surgical and nonsurgical peri-

\footnotetext{
*Correspondence to: Hyun-Jung Kim

Professor, Institute for Evidence-based Medicine, Department of Preventive Medicine, College of Medicine, Korea University, 73 Inchon-ro, Seongbuk-gu, Seoul, 02841, Republic of Korea

Tel: +82-2-2286-1341, Fax: +82-2-2286-1342, E-mail: moole@korea.ac.kr *Correspondence to: Jung-Chul Park

Assistant Professor, Department of Periodontology, College of Dentistry, Dankook University, 119 Dandae-ro, Dongnam-gu, Cheonan, 31116, Republic of Korea Tel: +82-41-550-0261, Fax: +82-303-3442-7364, E-mail: jcp@dent.dku.edu Received: January 6, 2017/Last Revision: March 6, 2017/Accepted: March 9, 2017
}

odontal therapies are effective against periodontitis by removing pathogenic dental plaque and calculus. ${ }^{5}$ However, some patients do not respond to conventional periodontal therapy ${ }^{6}$ or show highly elevated susceptibility to periodontal infection. ${ }^{7}$ Investigations of the mechanism underlying these phenomena have revealed that the immune response of subjects appears to play a critical role in the development and manifestation of periodontal diseases. ${ }^{8,9}$

In order to provide a better treatment modality to

Copyright@ 2017 The Korean Academy of Stomatognathic Function and Occlusion. (c) It is identical to Creative Commons Non-Commercial License. 
patients who are highly susceptible to periodontal diseases, host modulation using various therapeutic agents targeting the manipulation of the inflammatory pathway has been proposed as an adjunctive treatment with conventional periodontal therapy. ${ }^{10-13}$ Nonsteroidal anti-inflammatory drugs (NSAIDs) are among the most potent agents due to their direct inhibition of cyclooxygenase, which is responsible for the production of arachidonic acid metabolites. These metabolites are involved in tissue breakdown in periodontal diseases ${ }^{14}$ via the activation of the secretion of matrix metalloproteinase and osteoclasts. NSAIDs are therefore expected to have a strong efficacy for host modulation in patients with periodontal diseases.

In spite of the effectiveness of NSAIDs in host modulation, few systematic literature reviews have attempted to fully evaluate their impact in periodontal therapies, although it has been well established that the therapeutic effect of a host modulation agent can be synergistic when it is coupled to a cause-related periodontal therapy. ${ }^{15-17}$

Therefore, the aim of this review was to compare the effects of adjunctive use of NSAIDs with nonsurgical periodontal treatment (NSPT) on periodontal disease based on the available literature. Following focused question was addressed: "In patients with periodontal disease, is there an additional benefit of the oral administration of NSAIDs adjunctive with NSPT compared to control in terms of the clinical attachment level (CAL), probing depth (PD), and adverse outcomes in randomized controlled trials?"

\section{Materials and Methods}

\section{Data and literature source}

Relevant studies in electronic databases were identified by searching MEDLINE (from January 1, 1976 to March 11, 2015), EMBASE (from January 1, 1985 to March 11, 2015), and the Cochrane Central Register of Controlled Trials (from January 1, 1987 to March 11, 2015) without any restriction on language according to the Cochrane review methods. ${ }^{18}$ A search strategy was developed by combining text words and MeSH terms for MEDLINE and adapted to other databases (supplementary Appendix 1). In addition, key articles on periodontology published in the Journal of Periodontology, Journal of Clinical Periodontology, or Journal of Periodontal Research as well as the gray literature from January 1, 2005 to March 11, 2015 were searched manually for any missed relevant articles. ${ }^{19}$

\section{Inclusion criteria and study selection}

To be eligible for inclusion in our review, the studies had to fulfill the following criteria:

1. Randomized controlled trials that compared the effects of NSAIDs and NSPT versus NSPT only.

2. Subjects diagnosed with one of the classified types of periodontal disease, ${ }^{20}$ with the exception of gingival diseases and periodontitis associated with endodontic lesions.

3. All drugs administered via the oral route.

4. NSPT performed in both the NSAIDs and control groups.

5. Outcome included at least one clinical parameter of CAL or PD.

\section{Data extraction and analysis}

Studies to be included were selected independently by two reviewers (D.Y.K. and J.C.P.). After removing duplicates, suitable studies were identified by applying screening at the title/abstract level and then by reading the full texts. The level of agreement between reviewers in the screening process was assessed using Cohen's kappa coefficient. ${ }^{21}$ Data of identified studies were extracted independently by two reviewers using a prefabricated data extraction form. Disagreement was discussed by two reviewers, and if not resolved it was mediated by taking advice from the other authors. The methodological quality of each included study was assessed independently using the Cochrane risk-of-bias assessment tool. ${ }^{22}$ Suggested protocols were searched as part of the evaluation of reporting bias. ${ }^{23}$ To describe characteristics of the studies, data about subject populations 
and experimental designs were collected. The mean and standard deviation (SD) values of the changes in CAL and PD were collected for each group at baseline and the time of observation. If there were ambiguous or incomplete data, we contacted the authors by e-mail. The differences in the CAL gain and PD reduction between groups were calculated as mean and $95 \%$ confidence interval values. The results of the quantitative analysis were checked using both random-effect and fixed-effect models. To quantify heterogeneity, the $I^{2}$ statistic was used to estimate the proportion of inconsistency from true differences. ${ }^{24}$ Review Manager software (v.5.2, Nordic Cochrane Center, Copenhagen, Denmark) was used to perform the statistical tests.

\section{Results}

\section{Identification of studies}

The search of the databases initially identified 712 articles (Fig. 1), of which 287 duplicated articles were removed. At title/abstract screening, 400 articles that clearly were not consistent with the inclusion criteria were excluded. Reading the full texts of the remaining 25 articles resulted in the identification of 12 potentially relevant studies. The reasons for excluding 13 articles are tabulated in supplementary Appendix 2. The agreement between the two reviewers throughout the screening process was rated as good, with a Cohen's kappa coefficient of 0.69.

\section{Study characteristics and patient populations}

The demographic and experimental characteristics of the included studies are summarized in Table 1. There were differences between the studies in the kind of NSAIDs, daily dose, administration period, and observation period. The examined area, type of tooth, and number of data points per subject also varied among the studies. Treatment outcomes were presented using various outcome variables. For most of the studies, CAL or PD was reported as mean \pm $\mathrm{SD}$ values at the subject-level observation unit. ${ }^{25-33}$

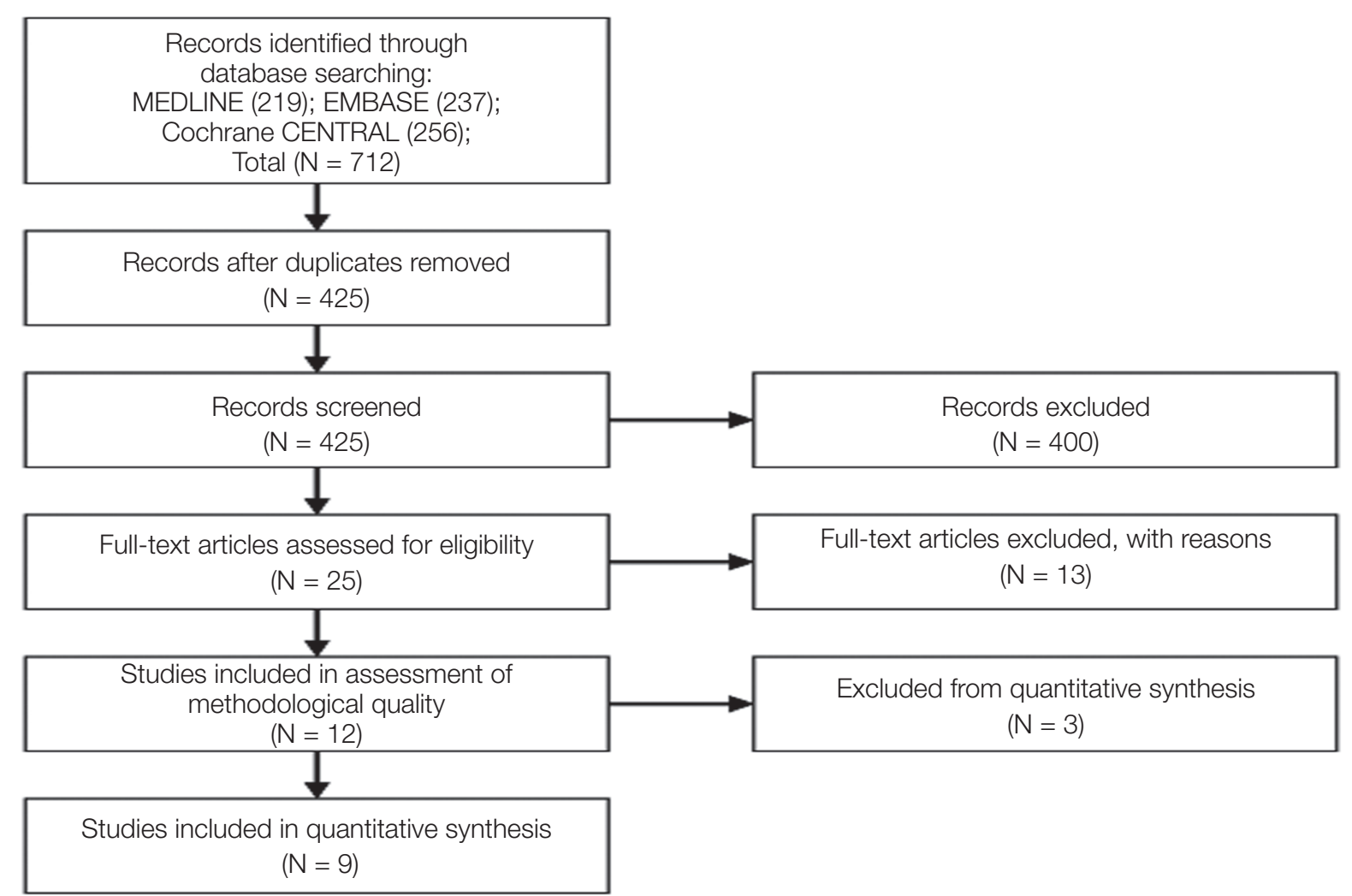

Fig. 1. Flow diagram. 
Table 1. Characteristics of included studies

\begin{tabular}{|c|c|c|c|c|c|}
\hline Study & Methods & Participants & Interventions & Clinical outcomes & Notes \\
\hline $\begin{array}{l}\text { Aras } \\
2007\end{array}$ & $\begin{array}{l}\text { RCT } \\
\text { Sample size at } \\
\text { entry: naproxen: } \\
\text { 17, placebo: } 17 \\
\text { Study duration: } 6 \\
\text { weeks } \\
\text { Unit of observation: } \\
\text { subject }\end{array}$ & $\begin{array}{l}\text { Age (mean } \pm \text { SD, yrs): } \\
36.24 \pm 2.4 \\
\text { Sex } \% \text { F: } 50 \% \text {, not } \\
\text { mentioned in each group } \\
\text { Smoker: excluded } \\
\text { DM: excluded }\end{array}$ & $\begin{array}{l}\mathrm{Rx} \text { ) naproxen } 275 \mathrm{mg} \\
1 \mathrm{~T} \text { for } 6 \text { weeks } \\
\text { Ctrl) placebo for } 6 \\
\text { weeks } \\
\text { Com) supragingival } \\
\text { scaling, OHI at baseline }\end{array}$ & $\begin{array}{l}\text { Subject based: } \\
\text { mean } \pm \text { SD of PD, GI, PI, } \\
\text { gingival bleeding index at } \\
\text { baseline and } 6 \text { weeks (full } \\
\text { mouth) } \\
\text { mean } \pm \text { SD of PD, GI, } \\
\text { PI, gingival bleeding index } \\
\text { at baseline and } 6 \text { weeks } \\
\text { (sample site) }\end{array}$ & $\begin{array}{l}\text { Probing site: full mouth } \\
\text { Sampling sites: two } \\
\text { deepest periodontal } \\
\text { pockets in the maxilla } \\
\text { incisors, canines, and } \\
\text { premolar } \\
\text { No. of probing sites: } \\
\text { not mentioned }\end{array}$ \\
\hline $\begin{array}{l}\text { Azoubel } \\
2008\end{array}$ & $\begin{array}{l}\text { RCT } \\
\text { Sample size at } \\
\text { entry: etoricoxib: } \\
\text { 11, placebo: } 10 \\
\text { Study duration: } 30 \\
\text { days } \\
\text { Unit of observation: } \\
\text { subject, site }\end{array}$ & $\begin{array}{l}\text { Patient: AP, age of } 18 \text { - } 35 \\
\text { years, had } \geq 20 \text { teeth, had } \\
\geq 4 \text { sites in different teeth } \\
\text { showing PD of } \geq 4 \mathrm{~mm}, \\
\text { and had } \geq 2 \text { sites showing } \\
\text { PD of } \geq 7 \mathrm{~mm} \\
\text { Age (mean } \pm \mathrm{SD} \text {, yrs): } \\
\text { etoricoxib: } 32.4 \pm 6.5, \\
\text { placebo: } 34.6 \pm 7.6 \\
\text { Sex } \% \text { F: etoricoxib: } 91 \% \text {, } \\
\text { placebo: } 90 \% \\
\text { Smoker: excluded } \\
\text { DM: excluded }\end{array}$ & $\begin{array}{l}\text { Rx) etoricoxib } 120 \mathrm{mg} \\
1 \mathrm{~T} \text { for } 7 \text { days } \\
\text { Ctrl) placebo } 1 \mathrm{~T} \text { for } 7 \\
\text { days } \\
\text { Com) OHI and SRP } \\
\text { during drug intake, } \\
\text { weekly oral hygiene } \\
\text { reinforcement and } \\
\text { prophylaxis until } 30 \\
\text { days }\end{array}$ & $\begin{array}{l}\text { Subject-based: mean } \pm \\
\text { SD of PD, CAL, gingival } \\
\text { recession, visual plaque } \\
\text { index, and BOP at baseline } \\
\text { and } 30 \text { days } \\
\text { Site-based: frequency ( } \%) \\
\text { of sites showing CAL gain } \\
\text { of } \geq 2 \mathrm{~mm} \\
\text { Dropout and reason: } \\
\text { Rx) } 1 \text {, lost to follow- } \\
\text { up (did not return for } \\
\text { reassessment) }\end{array}$ & $\begin{array}{l}\text { Probing site: sites } \\
\text { showing baseline PD } \\
\text { of }>3 \mathrm{~mm} \text { only and } \\
\text { gingival increase was } \\
\text { excluded } \\
\text { Sampling sites: deepest } \\
\text { periodontal pockets in } \\
\text { the maxilla anterior and } \\
\text { premolar } \\
\text { No. of probing sites: } 6\end{array}$ \\
\hline $\begin{array}{l}\text { Buduneli } \\
2010\end{array}$ & $\begin{array}{l}\text { RCT } \\
\text { Sample size at } \\
\text { entry: meloxicam: } \\
\text { 26, placebo: } 24 \\
\text { Study duration: } 4 \\
\text { weeks } \\
\text { Experimental unit: } \\
\text { subject }\end{array}$ & $\begin{array}{l}\text { Patient: CP, Caucasian, had } \\
\geq 4 \text { sites showing CAL } \\
\text { of } \geq 4 \mathrm{~mm} \text { and PD of } \geq \\
5 \mathrm{~mm} \text { and two of which } \\
\text { were in the anterior region } \\
\text { Age (mean } \pm \text { SD, yrs): } \\
\text { meloxicam: } 48.4 \pm 2.1, \\
\text { placebo: } 47.2 \pm 1.9 \\
\text { Sex } \% \text { F: meloxicam } 50 \%, \\
\text { placebo } 50 \% \\
\text { Smoking: excluded } \\
\text { DM: excluded }\end{array}$ & $\begin{array}{l}\mathrm{Rx} \text { ) meloxicam } 7.5 \mathrm{mg} \\
1 \mathrm{~T} \text { for } 10 \text { days } \\
\text { Ctrl) placebo for } 10 \\
\text { days } \\
\text { Com) SRP on day } 3 \text { of } \\
\text { drug intake }\end{array}$ & $\begin{array}{l}\text { Subject-based: mean } \pm \text { SD } \\
\text { of PD, CAL, dichotomous } \\
\text { plaque index (present } \\
\text { or absent), and papilla } \\
\text { bleeding index (PBI) at } \\
10 \text { days and } 4 \text { weeks after } \\
\text { SRP } \\
\text { Dropout and reason: Rx) } \\
\text { 3, Ctrl) 2, discontinued } \\
\text { study }\end{array}$ & $\begin{array}{l}\text { Probing, sampling } \\
\text { sites: } 2 \text { sampling sites } \\
\text { of the single-root teeth } \\
\text { showing PD of } \geq 5 \\
\text { mm, CAL of } \geq 4 \mathrm{~mm}, \\
\text { and BOP }(+) \\
\text { No. of probing sites per } \\
\text { tooth: } 2 \text { (mesiobuccal, } \\
\text { distobuccal) }\end{array}$ \\
\hline $\begin{array}{l}\text { Jeffcoat } \\
1991\end{array}$ & $\begin{array}{l}\text { RCT } \\
\text { Sample size at } \\
\text { entry: naproxen: } 7 \text {, } \\
\text { placebo: } 8 \\
\text { Study duration: } 3 \\
\text { months } \\
\text { Unit of observation: } \\
\text { subject }\end{array}$ & $\begin{array}{l}\text { Patient: AP, had } \geq 5 \text { sites } \\
\text { showing radiographic } \\
\text { evidence of rapidly } \\
\text { progressive periodontitis, } \\
\text { age }<35 \text {, and had } \geq 20 \\
\text { teeth } \\
\text { Age (range, yrs): } 18-41 \\
\text { Sex F \%: overall } 73 \% \text {, no } \\
\text { detail per group } \\
\text { Smoking: not mentioned } \\
\text { DM: excluded }\end{array}$ & $\begin{array}{l}\text { Rx) naproxen } 1000 \mathrm{mg} \\
\text { per day for } 3 \text { months } \\
\text { Ctrl) placebo for } 3 \\
\text { months } \\
\text { Com) SRP } 2 \text { weeks } \\
\text { before drug intake, } \\
\text { OHI } 2 \text { weeks before } \\
\text { drug intake and after } \\
\text { 1, 2, } 3 \text { months of drug } \\
\text { intake }\end{array}$ & $\begin{array}{l}\text { Subject-based: mean } \pm \text { SD } \\
\text { of the change in CAL and } \\
\text { GI between baseline and } 3 \\
\text { months } \\
\text { Site-based: radiographic: } \\
\text { frequency (\%) of tooth } \\
\text { showing increased, no } \\
\text { change, or decreased bone }\end{array}$ & $\begin{array}{l}\text { No. probing sites: } \\
\text { five test sites which } \\
\text { had evidence of } \\
\text { rapidly progressive } \\
\text { periodontitis }\end{array}$ \\
\hline $\begin{array}{l}\mathrm{Ng} \text { and } \\
\text { Bissada } \\
1998\end{array}$ & $\begin{array}{l}\text { RCT } \\
\text { Split mouth design } \\
\text { (SRP / no SRP) } \\
\text { Sample size at } \\
\text { entry: no detail } \\
\text { (32 subjects were } \\
\text { randomly assigned } \\
\text { to one of the } 4 \\
\text { treatment group) } \\
\text { Study duration: } 24 \\
\text { weeks } \\
\text { Unit of observation: } \\
\text { subject }\end{array}$ & $\begin{array}{l}32 \text { subjects with } \\
\text { generalized periodontitis } \\
\text { that radiographic bone } \\
\text { loss is greater than } 50 \% \text { in } \\
\text { at least } 2 \text { matching tooth } \\
\text { types per subject, PD of } \geq \\
5 \text { mm in } 1 \text { or more sites in } \\
\text { at least } 2 \text { matching types } \\
\text { Age (range, yrs): } 32-72 \text { yrs } \\
\text { Sex F \%: overall } 44 \% \text {, no } \\
\text { detail per group } \\
\text { Smoking: smoker }=15 \text {, } \\
\text { non-smoker }+ \text { former } \\
\text { smoker }=17 \\
\text { DM: excluded }\end{array}$ & $\begin{array}{l}\text { Rx1) doxycycline } 200 \\
\text { mg (first day), } 100 \mathrm{mg} \\
\text { (other day) per day for } \\
6 \text { weeks } \\
\text { Rx2) ibuprofen } 800 \mathrm{mg} \\
\text { per day for } 6 \text { weeks } \\
\text { Rx3) doxycycline }+ \\
\text { ibuprofen for } 6 \text { weeks } \\
\text { Ctrl) placebo for } 6 \\
\text { weeks } \\
\text { Com) SRP at baseline }\end{array}$ & $\begin{array}{l}\text { Subejct-based: mean } \pm \\
\text { SD of PI, GI, PD, CAL at } \\
\text { baseline, 3, 6, 9, 12, and } 24 \\
\text { weeks }\end{array}$ & $\begin{array}{l}\text { Probing sites: full } \\
\text { mouth } \\
\text { No. of probing sites } \\
\text { per tooth: } 6 \text { (using pre- } \\
\text { fabricated stents) }\end{array}$ \\
\hline
\end{tabular}


Table 1. (Continued) Characteristics of included studies

\begin{tabular}{|c|c|c|c|c|c|}
\hline Study & Methods & Participants & Interventions & Clinical outcomes & Notes \\
\hline $\begin{array}{l}\text { Özgören } \\
2014\end{array}$ & $\begin{array}{l}\text { RCT } \\
\text { Sample size at } \\
\text { entry: tenoxicam: } \\
\text { 16, placebo: } 16 \\
\text { Study duration: } 30 \\
\text { days } \\
\text { Unit of observation: } \\
\text { subject }\end{array}$ & $\begin{array}{l}\text { Patient: } \mathrm{CP}, \text { had } \geq 4 \\
\text { periodontal sites showing } \\
\text { PD of } 4 \text { - } 6 \mathrm{~mm} \text { and } \\
\text { radiographic evidence of } \\
\text { bone and attachment loss } \\
\text { involving the maxillary } \\
\text { anterior teeth } \\
\text { Age (mean } \pm \text { SD yrs): } \\
\text { tenoxicam: } 40.9 \pm 8.2 \text {, } \\
\text { placebo: } 42.3 \pm 7.3 \\
\text { Sex F \%: overall } 44 \%, \text { no } \\
\text { details per group } \\
\text { Smoking: excluded } \\
\text { DM: excluded }\end{array}$ & $\begin{array}{l}\text { Rx) tenoxicam } 20 \mathrm{mg} \\
\text { per day for } 10 \text { days } \\
\text { Ctrl) placebo for } 10 \\
\text { days } \\
\text { Com) SRP at baseline, } \\
\text { OHI before baseline } \\
\text { and at baseline }\end{array}$ & $\begin{array}{l}\text { Subject-based: mean } \pm \text { SD } \\
\text { of PI, GI, PD, gingival } \\
\text { bleeding time index and } \\
\text { CAL at baseline and } 30 \\
\text { days }\end{array}$ & $\begin{array}{l}\text { Probing sites: } 4 \text { teeth } \\
\text { (PD of } 4-6 \text { mm } \\
\text { and radiographic } \\
\text { evidence of bone } \\
\text { and attachment loss } \\
\text { involving the maxillary } \\
\text { anterior teeth) } \\
\text { No. of probing sites } \\
\text { per tooth: } 6\end{array}$ \\
\hline $\begin{array}{l}\text { Pinho } \\
\text { Mde } \\
2008\end{array}$ & $\begin{array}{l}\text { RCT } \\
\text { Sample size at } \\
\text { entry: loxoprofen } \\
\text { 30, placebo: } 30 \\
\text { Study duration: } 28 \\
\text { days } \\
\text { Unit of observation: } \\
\text { subject }\end{array}$ & $\begin{array}{l}\text { Patient: periodontitis, had } \\
\geq 20 \text { teeth, } 35 \text { - } 0 \text { years, } \\
\text { had } \geq 2 \text { teeth showing } \\
\text { CAL of } \geq 6 \mathrm{~mm} \text {, and had } \\
\geq 1 \text { site showing PD of } \geq \\
5 \mathrm{~mm} \\
\text { Age (mean } \pm \text { SD): } \\
\text { loxoprofen } 41 \pm 4, \\
\text { placebo: } 42 \pm 5 \\
\text { Sex F\%: loxoprofen } 53 \%, \\
\text { placebo: } 53 \% \\
\text { Smoking: excluded } \\
\text { DM: excluded }\end{array}$ & $\begin{array}{l}\text { Rx) loxoprofen } 60 \mathrm{mg} \\
\text { per day for } 28 \text { days } \\
\text { Ctrl) placebo for } 28 \\
\text { days } \\
\text { Com) SRP of half } \\
\text { mouth at baseline and } \\
\text { contra-lateral side on } \\
\text { day } 14 \text { and OHI at } \\
\text { baseline and } 14 \text { days }\end{array}$ & $\begin{array}{l}\text { Subject-based: PD, } \% \\
\text { BOP, and PI at baseline } \\
\text { (demographic data) } \\
\text { Site-based: } \% \text { sites } \\
\text { showing PD of }<4,4-7 \text {, } \\
\text { and } \geq 7 \mathrm{~mm} \text { at baseline, } \\
14 \text {, and } 28 \text { days }\end{array}$ & $\begin{array}{l}\text { Probing sites: full } \\
\text { mouth } \\
\text { No. of probing sites } \\
\text { per tooth: } 6\end{array}$ \\
\hline $\begin{array}{l}\text { Reddy } \\
1993\end{array}$ & $\begin{array}{l}\text { RCT } \\
\text { Sample size at } \\
\text { entry: overall } 27, \\
\text { no details per } \\
\text { group } \\
\text { Study duration: } 28 \\
\text { days } \\
\text { Unit of observation: } \\
\text { subject, site }\end{array}$ & $\begin{array}{l}\text { Patient: had a history } \\
\text { of periodontitis prior to } \\
\text { the age of } 35 \text {, had } \geq 3 \\
\text { posterior teeth in each } \\
\text { of } 3 \text { quadrants showing } \\
\text { alveolar bone loss of } 30 \\
-50 \% \text {, and had } \geq 3 \text { sites } \\
\text { showing active bone loss } \\
\text { as determined by bone } \\
\text { scan } \\
\text { Age (mean } \pm \mathrm{SD}, \mathrm{yrs} \text { ): } \\
\text { overall: } 36.5 \pm 7.88, \text { no } \\
\text { details per group } \\
\text { Sex F } \%: \text { overall } 68 \% \\
\text { Smoking: not mentioned } \\
\text { DM: excluded }\end{array}$ & $\begin{array}{l}\text { Rx1) meclofenamate } \\
100 \text { mg per day for } 6 \\
\text { months } \\
\text { Rx2) meclofenamate } \\
200 \text { mg per day for } 6 \\
\text { months } \\
\text { Ctrl) placebo for } 6 \\
\text { months } \\
\text { Com) OHI, SRP, and } \\
\text { occlusal adjustment } \\
\text { (when needed) before } 2 \\
\text { - } 4 \text { weeks from baseline } \\
\text { SRP at } 3 \text { months and } 6 \\
\text { months }\end{array}$ & $\begin{array}{l}\text { Subject-based: GI, PI, PD } \\
\text { and CAL at baseline, } 3 \\
\text { months, and } 6 \text { months } \\
\text { Site-based: CAL at } \\
\text { baseline to } 6 \text { months } \\
\text { Dropout and reason: } \\
\text { Rx1) } 3 \text { (2: gastrointestinal } \\
\text { irritation, 1: disliked } \\
\text { medication) } \\
\text { Rx2) } 1 \text { (disliked } \\
\text { medication) } \\
\text { Ctrl) } 1 \text { (military service) } \\
\text { Adverse effect } \\
\text { gastrointestinal irritation }\end{array}$ & $\begin{array}{l}\text { Probing sites: } 6-8 \\
\text { teeth (which show } \\
\text { high bone-seeking } \\
\text { radiopharmaceutical } \\
\text { uptake ratio) } \\
\text { No. of probing } \\
\text { sites per tooth: not } \\
\text { mentioned } \\
\text { Statistics were } \\
\text { expressed as mean } \pm \\
\text { standard error }\end{array}$ \\
\hline $\begin{array}{l}\text { Shiloah } \\
2014\end{array}$ & $\begin{array}{l}\text { RCT } \\
\text { Sample size at } \\
\text { entry: aspirin: } 18, \\
\text { placebo } 14 \\
\text { Study duration: } 12 \\
\text { months } \\
\text { Unit of observation: } \\
\text { subject }\end{array}$ & $\begin{array}{l}\text { Patient: chronic } \\
\text { periodontitis, self-reported } \\
\text { smoker, had } \geq 4 \text { teeth } \\
\text { showing PD of } 5 \mathrm{~mm} \text { and } \\
\text { attachment loss of }>2 \\
\text { mm } \\
\text { Age (mean } \pm \text { SD yrs): } \\
\text { aspirin: } 53.25 \pm 7.31 \text {, } \\
\text { placebo: } 48.83 \pm 8.34 \\
\text { Sex F } \%: 58 \% \text { except } \\
\text { dropout, not mentioned at } \\
\text { entry } \\
\text { Smoking: } 100 \% \text { (only } \\
\text { smoker included) } \\
\text { DM: excluded }\end{array}$ & $\begin{array}{l}\mathrm{Rx} \text { ) aspirin } 325 \mathrm{mg} \text { per } \\
\text { day for a year } \\
\text { Ctrl) placebo for a year } \\
\text { Com) extraction of } \\
\text { "hopeless", non- } \\
\text { restorable tooth, OHI, } \\
\text { SRP before baseline }\end{array}$ & $\begin{array}{l}\text { Subject-based: } \\
\% \text { sites plaque index }=2 \\
\text { and } 3 \text {, } \\
\% \text { sites GI }=0,1 \text {, and } 2 \text {, } \\
\% \text { sites PD } 1-3,4 \text { - } 6 \text {, and } \\
\geq 7 \mathrm{~mm} \text {, } \\
\% \text { sites PAL } 0-2,3-4 \text {, } \\
\text { and }>4 \mathrm{~mm} \text {, } \\
\% \text { sites BOP at baseline, } 3 \text {, } \\
6,9 \text {, and } 12 \text { months } \\
\text { Dropout and reason } \\
\text { Rx) } 6 \text { (lost to follow up, } \\
\text { e.g., job schedule change, } \\
\text { moved) } \\
\text { Ctrl) } 2 \text { (lost to follow up } \\
\text { e.g., job schedule change, } \\
\text { moved) }\end{array}$ & $\begin{array}{l}\text { Probing sites: full } \\
\text { mouth } \\
\text { No. of probing } \\
\text { sites per tooth: not } \\
\text { mentioned }\end{array}$ \\
\hline
\end{tabular}


Table 1. (Continued) Characteristics of included studies

\begin{tabular}{|c|c|c|c|c|c|}
\hline Study & Methods & Participants & Interventions & Clinical outcomes & Notes \\
\hline $\begin{array}{l}\text { Taiyep } \\
\text { Ali and } \\
\text { Waite } \\
1993\end{array}$ & $\begin{array}{l}\text { RCT } \\
\text { Sample size at } \\
\text { entry: overall } 17, \\
\text { no details per } \\
\text { group } \\
\text { Study duration: } 8 \\
\text { weeks } \\
\text { Unit of observation: } \\
\text { subject }\end{array}$ & $\begin{array}{l}\text { Patient: chronic } \\
\text { periodontitis, had } 6 \\
\text { Ramfjord teeth with mild } \\
\text { to severe bone loss } \\
\text { Age (range yrs): overall } 28 \\
\text { - } 40 \\
\text { Sex F \%: overall: } 71 \% \text {, no } \\
\text { details per group } \\
\text { Smoking: not mentioned } \\
\text { DM: excluded }\end{array}$ & $\begin{array}{l}\text { Rx) ibuprofen } 800 \mathrm{mg} \\
\text { per day for } 14 \text { days } \\
\text { Ctrl) no medication } \\
\text { Com) OHI, SRP (split } \\
\text { mouth) at baseline; } \\
\text { OHI, oral prophylaxis, } \\
\text { and re-evaluation at 2, } \\
4,6 \text { and } 8 \text { weeks }\end{array}$ & $\begin{array}{l}\text { Subject-based: PD, gingival } \\
\text { color index, and gingival } \\
\text { bleeding at } 2,4,6 \text {, and } 8 \\
\text { weeks } \\
\text { Dropout and reason: Rx) } \\
\text { 2, unknown }\end{array}$ & $\begin{array}{l}\text { Probing sites: } 6 \text { teeth } \\
\text { (Ramfjord teeth) } \\
\text { No. of probing sites } \\
\text { per tooth: } 6 \\
\text { No placebo medication }\end{array}$ \\
\hline $\begin{array}{l}\text { Vardar } \\
2003\end{array}$ & $\begin{array}{l}\text { RCT } \\
\text { Sample size at } \\
\text { entry: nimesulide } \\
\text { 10, naproxen } 10 \text {, } \\
\text { and placebo } 10 \\
\text { Study duration: } 3 \\
\text { months } \\
\text { Unit of observation: } \\
\text { subject }\end{array}$ & $\begin{array}{l}\text { Patient: CP, had } \geq 18 \\
\text { teeth, and had } \geq 2 \text { teeth } \\
\text { in each quadrant showing } \\
\text { PD of } \geq 5 \mathrm{~mm} \text { and CAL } \\
\text { of } \geq 4 \mathrm{~mm} \\
\text { Age (mean } \pm \mathrm{SD}, \mathrm{yrs} \text { ): } \\
\text { nimesulide: } 46.6 \pm 14.1, \\
\text { naproxen: } 44.0 \pm 3.6, \\
\text { placebo: } 43.9 \pm 6.9 \\
\text { Sex F } \%: \text { nimesulide: } 40 \% \text {, } \\
\text { naproxen: } 30 \% \text {, placebo: } \\
\text { 50\% } \\
\text { Smoker: not excluded } \\
\text { DM: excluded }\end{array}$ & $\begin{array}{l}\text { Rx1) nimesulide } 200 \\
\text { mg per day for } 10 \text { days } \\
\text { Rx2) naproxen } 550 \mathrm{mg} \\
\text { per day for } 10 \text { days } \\
\text { Ctrl) placebo for } 10 \\
\text { days } \\
\text { Com) OHI at baseline, } \\
\text { SRP at } 3 \text { days }\end{array}$ & $\begin{array}{l}\text { Subejct-based: } \\
\text { CAL and PD at baseline } \\
\text { and } 3 \text { months } \\
\text { PI, PBI at baseline, } 10 \\
\text { days, and } 3 \text { months }\end{array}$ & $\begin{array}{l}\text { Probing sites: } 4 \text { sites } \\
\text { (2 sampling sites } x \\
2 \text { approximal tooth } \\
\text { sites adjacent to the } \\
\text { sampling site) }\end{array}$ \\
\hline $\begin{array}{l}\text { Yen } \\
2008\end{array}$ & $\begin{array}{l}\text { RCT } \\
\text { Sample size at } \\
\text { entry: overall } 131 \\
\text { Study duration: } 12 \\
\text { months } \\
\text { Unit of observation: } \\
\text { site }\end{array}$ & $\begin{array}{l}\text { Patient: CP, had }>16 \text { teeth } \\
\text { (at least two of which } \\
\text { were molars), had } \geq 4 \\
\text { teeth showing PD of }>4 \\
\text { mm and CAL of }>2 \mathrm{~mm} \text {, } \\
\text { and had } \geq 2 \text { interproximal } \\
\text { areas showing radiographic } \\
\text { evidence of bone loss } \\
\text { Age (mean } \pm \mathrm{SD} \text {, yrs): } \\
\text { overall } 48.6 \pm 9.9 \\
\text { Sex F } \%: 47 \% \text { at } 3 \text { months } \\
\text { Smoker: } 34.7 \% \text { (former } \\
\text { smoker: } 23.8 \%, \\
\text { nonsmoker: } 39.6 \% \text { ) } \\
\text { DM: not excluded }\end{array}$ & $\begin{array}{l}\text { Rx) celecoxib } 200 \mathrm{mg} \\
\text { per day for } 3 \text { months } \\
\text { Ctrl) placebo for } 3 \\
\text { months } \\
\text { Com) SRP at baseline } \\
(1 / 2) \text { and within } 1 \text { - } 2 \\
\text { weeks }(1 / 2) \\
\text { OHI at baseline, } 3,6,9 \text {, } \\
\text { and } 12 \text { months } \\
\text { oral prophylaxis at } 3,6 \text {, } \\
9 \text {, and } 12 \text { months }\end{array}$ & $\begin{array}{l}\text { Site-based } \\
\text { CAL by BPD = } 1-3,4-6 \text {, } \\
\text { and } \geq 7 \mathrm{~mm} \\
\text { PD by BPD }=1-3,4-6 \text {, } \\
\text { and } \geq 7 \mathrm{~mm} \\
\% \text { sites showing CAL gain } \\
\geq 2 \mathrm{~mm} \\
\% \text { sites showing CAL loss } \\
\geq 2 \mathrm{~mm} \\
\text { Dropout and reason } \\
\text { Total } 66 \text { (highly publicized } \\
\text { finding that subjects } \\
\text { taking large dosages of } \\
\text { COX-2 inhibitors showed } \\
\text { an increased risk for } \\
\text { cardiovascular episodes) }\end{array}$ & $\begin{array}{l}\text { Probing sites: full } \\
\text { mouth } \\
\text { No. of probing sites } \\
\text { per tooth: } 6 \\
66 \text { subjects were } \\
\text { dropout }\end{array}$ \\
\hline
\end{tabular}

AP: aggressive periodontitis, BOP: bleeding on probing, BPD: baseline probing depth, CAL: Clinical attachment loss, Com): common intervention, CP: chronic periodontitis, Ctrl): control group, GI: gingival index, GCF: gingival crevicular fluid, DM: diabetes mellitus, OHI: oral hygiene instruction, PD: probing depth, PI: plaque index, Rx): nonsteroidal anti-inflammatory drugs group, SD: standard deviation, yrs: years, Sex \% F: percentage of female, SRP: scaling and root planing.

\section{Methodological quality of included studies}

The included studies were assessed using the Cochrane risk-of-bias assessment tool (Fig. 2, supplementary Appendix 3). Publication bias could not be assessed due to the small number of appropriate studies (7 for CAL and 8 for PD), since asymmetry of the funnel plot is generally only tested when at least 10 studies are included in a meta-analysis.

\section{Effects of interventions}

Data reported as mean $\pm \mathrm{SD}$ values with the subject-level observation unit pooled by observation time. The differences in the CAL gain and PD reduction between NSAIDs and control groups using a random-effect model are illustrated in Fig. 3 and Fig. 4 as mean and $95 \%$ confidence interval values. There was no significant difference in the mean CAL gain 
Random sequence generation (selection bias)

Allocation concealment (selection bias)

Blinding of participants and personnel (performance bias)

Blinding of outcome assessment (detection bias)

Incomplete outcome data (attrition bias)

Selective reporting (reporting bias)

Other bias

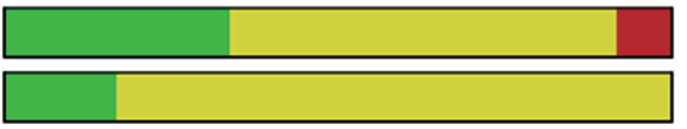

$$
\text { L }
$$

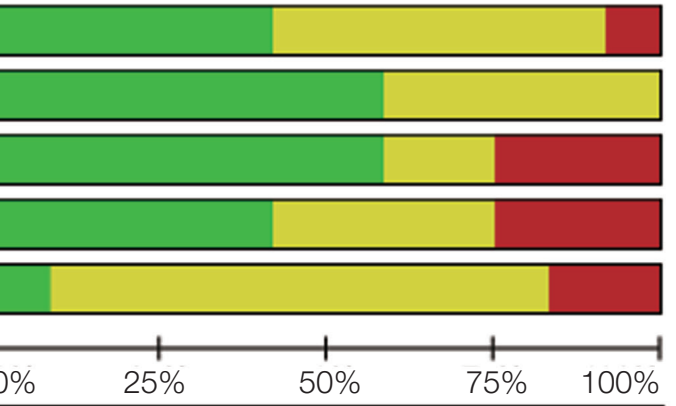

Low risk of bias

Uuclear risk of bias

High risk of bias

Fig. 2. Risk of bias graph: review authors' judgements about each risk of bias item presented as percentages across all included studies.

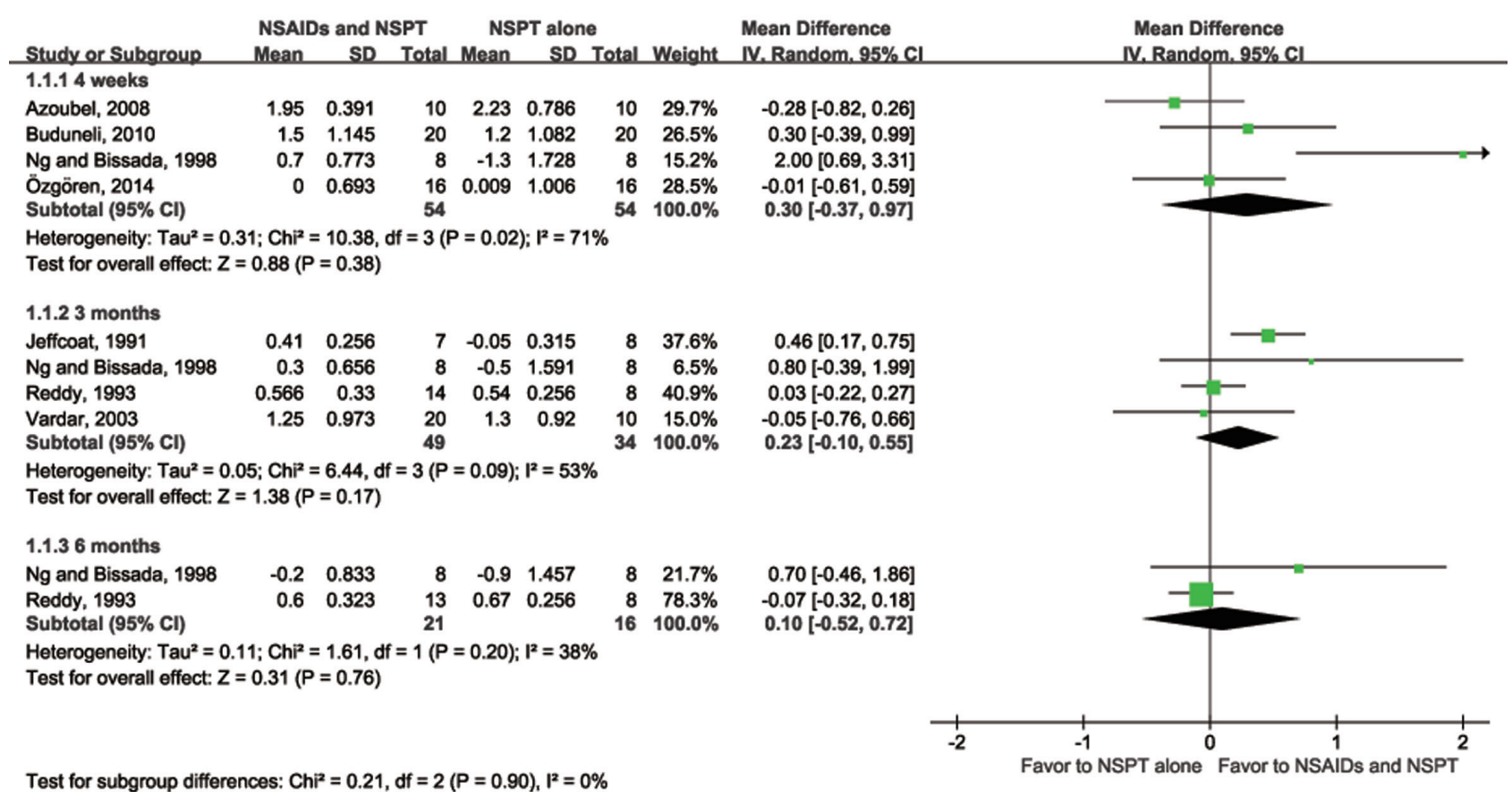

Fig. 3. Forest plot of changes in CAL for in randomized clinical trials that administrated NSAIDs adjunctive to nonsurgical periodontal treatment.

at any observation time (Fig. 3). A significant reduction in PD-with a mean difference of $0.34 \mathrm{~mm}(95 \%$ confidence interval $=0.29$ to 0.40$)$-was seen only at 6 weeks relative to the control group (Fig. 4).

The number of adverse reactions, the reasons for dropouts, and reports of adverse reactions are listed in Table 1. Reddy et al. ${ }^{31}$ reported that their dropouts were due to gastrointestinal discomfort in the group receiving meclofenamate at $200 \mathrm{mg} /$ day, but the reason for other dropouts was unknown in most cases (e.g., lost to follow-up or a discontinued study). 


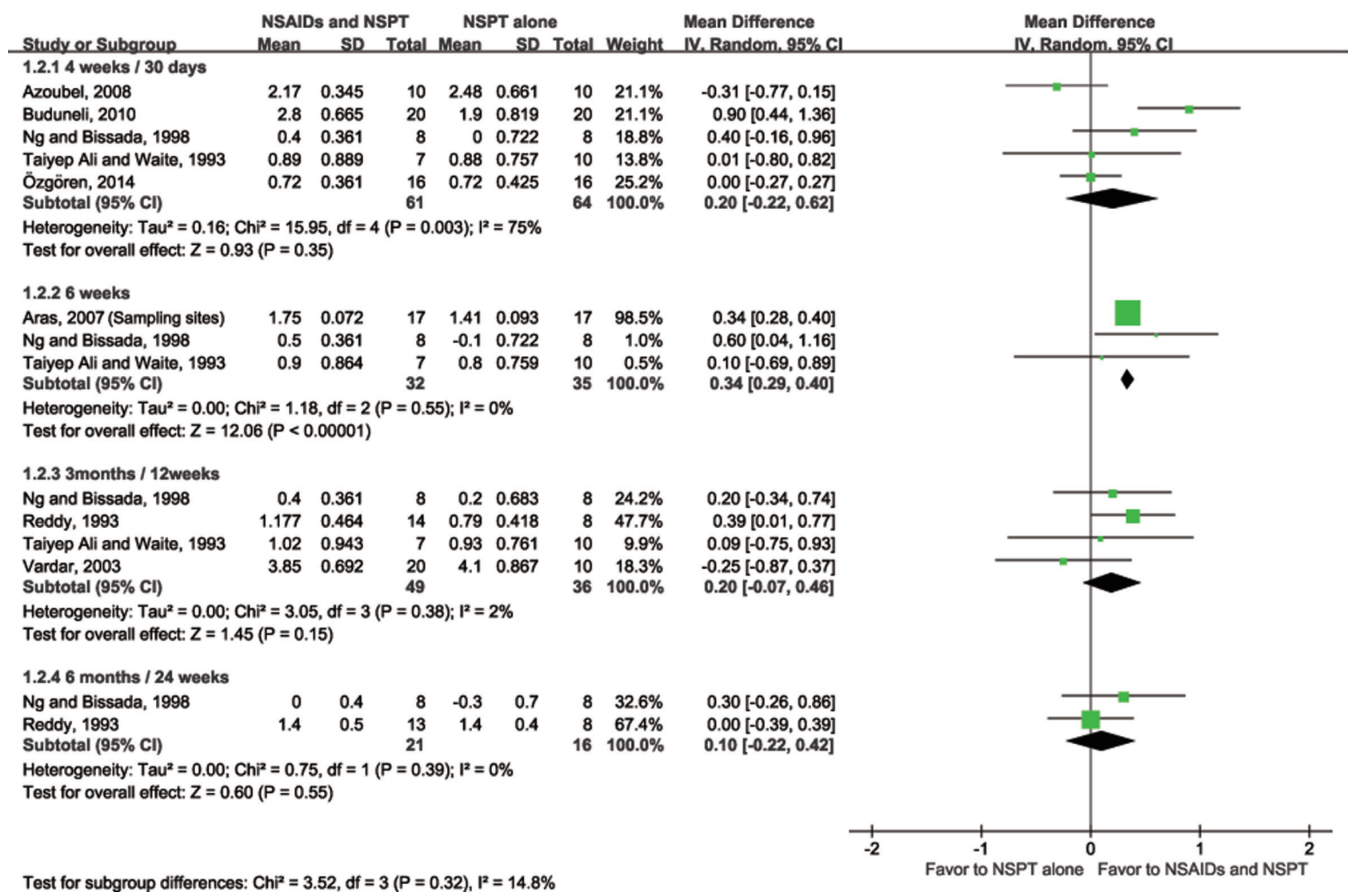

Fig. 4. Forest plot of changes in PD for in randomized clinical trials that administrated NSAIDs adjunctive to nonsurgical periodontal treatment.

\section{Discussion}

The studies included in this meta-analysis exhibited a substantial heterogeneity, which was due to a few reports of significantly higher mean differences in CAL gain or PD reduction in the NSAIDs group than the control group. For instance, Ng and Bissada ${ }^{29}$ reported a higher mean difference in the NSAIDs group at 6 weeks posttreatment, but this was due to a smaller CAL loss in the NSAIDs group compared to the control group, rather than to a superior CAL gain. Likewise, Buduneli et al. ${ }^{27}$ enrolled patients with a deeper PD at baseline in the NSAIDs group than the control group, and this may have resulted in a superior PD reduction considering that scaling and root planing reportedly produces a greater PD reduction in sites with a deeper PD. ${ }^{5,34}$

The following differences accounted for the heterogeneity. Firstly, subjects were recruited in each study using different inclusion criteria and exclusion criteria. Secondly, additional oral prophylaxis or oral hygiene instruction was either not planned or, if it was planned, the schedule varied among the studies. These additional procedures may produce an appropriately maintained plaque state in the enrolled patients, and they may have resulted in the treatment responses differing among the studies. In addition, the state of plaque deposition was not presented as a unified outcome variable. Moreover, kinds of NSAIDs the daily dose, administration period, and observation period of the regime varied among the studies. These differences may have contributed to 
the inconsistent results.

It is well known that NSAIDs may induce gastrointestinal irritation, and two of the patients who dropped out for this reason had received meclofenamate at $200 \mathrm{mg} /$ day for 6 months. ${ }^{31}$ To minimize gastrointestinal irritation and ulcers from NSAIDs, limiting the dose or co-administration with protonpump inhibitors or $\mathrm{H}_{2}$-receptor antagonist can be considered. ${ }^{35}$ It has been reported that NSAIDs can impair oral mucosal repair as well as gastrointestinal mucosa, ${ }^{36}$ so whether NSAIDs compromise gingival repair following NSPT also needs to be investigated.

A recent nationwide retrospective study failed to show any benefit of low-dose aspirin when comparing mean CAL and PD data from 2335 subjects in a national health and nutrition examination survey. ${ }^{37}$ In addition, systematic reviews have found no clear effect of NSAIDs on clinical outcomes. ${ }^{17}$ Our present results are in accordance with these previous findings. However, two reviews have found positive effects of NSAIDs on gingival inflammation and periodontal-disease progression by reducing the rate of alveolar bone resorption. ${ }^{38,39}$ These conflicting findings are probably due to the bone preservation effect of NSAIDs and it might not be reflected in clinical parameters including PD or CAL. Also, it would take a long time for bony changes to be measurable by periodontal probes.

The methodological quality of the included studies was not high due to the lack of information. In addition, there were problems associated with partial tooth recording (i.e., including only specific types of teeth), which has the risk of overestimating or underestimating the periodontal condition of patients. ${ }^{40}$ Not only an adequate number of subjects but also an adequate number of data points from different types of teeth are needed to accurately reflect the status of included subjects. Taking these factors into account, further randomized controlled trials are needed to achieve a high methodological quality, involving subject-level observation units and sufficient data points via full-mouth observations as well as the inclusion of sufficient subjects to achieve an adequate statistical power for detecting a clinically meaningful effect size of NSAIDs.

\section{Conclusion}

The present review found clinical benefit for NSAIDs and NSPT over NSPT alone in some administration periods. It is difficult to conclude definitively whether a specific regime is superior to others, although some studies have found significant benefits and the others do not, due to the smallness of the included samples and the substantial heterogeneity among studies.

\section{ORCID}

Dae-Young Kang http://orcid.org/0000-0002-4311-4118

In-Woo Cho http://orcid.org/0000-0003-4985-3816

Hyun-Seung Shin http://orcid.org/0000-0002-1410-9731

Hyeong-Sik Ahn http://orcid.org/0000-0002-2084-7466

Hyun-Jung Kim http://orcid.org/0000-0003-2018-2385

Jung-Chul Park http://orcid.org/0000-0002-2041-8047

\section{References}

1. Consensus report. Periodontal diseases: pathogenesis and microbial factors. Ann Periodontol 1996;1: 926-32.

2. Havemose-Poulsen A, Sørensen LK, Bendtzen K, Holmstrup P. Polymorphisms within the IL-1 gene cluster: effects on cytokine profiles in peripheral blood and whole blood cell cultures of patients with aggressive periodontitis, juvenile idiopathic arthritis, and rheumatoid arthritis. J Periodontol 2007; 78:475-92.

3. Löe H. Periodontal disease. The sixth complication of diabetes mellitus. Diabetes Care 1993;16:329-34.

4. Tomar SL, Asma S. Smoking-attributable periodontitis in the United States: findings from NHANES III. National Health and Nutrition Examination Survey. J Periodontol 2000;71:743-51.

5. Cobb CM. Clinical significance of non-surgical periodontal therapy: an evidence-based perspective of scaling and root planing. J Clin Periodontol 2002;29 Suppl 2:6-16.

6. Haffajee AD, Socransky SS, Ebersole JL. Survival analysis of periodontal sites before and after peri- 
odontal therapy. J Clin Periodontol 1985;12:553-67.

7. Giannobile WV, Braun TM, Caplis AK, DoucetteStamm L, Duff GW, Kornman KS. Patient stratification for preventive care in dentistry. J Dent Res 2013;92:694-701.

8. Kornman KS, Page RC, Tonetti MS. The host response to the microbial challenge in periodontitis: assembling the players. Periodontol 2000 1997;14: 33-53.

9. Page RC. The role of inflammatory mediators in the pathogenesis of periodontal disease. J Periodontal Res 1991;26(3 Pt 2):230-42.

10. Lane N, Armitage GC, Loomer P, Hsieh S, Majumdar S, Wang HY, Jeffcoat M, Munoz T. Bisphosphonate therapy improves the outcome of conventional periodontal treatment: results of a 12-month, randomized, placebo-controlled study. J Periodontol 2005;76:1113-22.

11. Leitão RF, Ribeiro RA, Chaves HV, Rocha FA, Lima V, Brito GA. Nitric oxide synthase inhibition prevents alveolar bone resorption in experimental periodontitis in rats. J Periodontol 2005;76:956-63.

12. Martinez GL, Koury JC, Martins MA, Nogueira F, Fischer RG, Gustafsson A, Figueredo CM. Serum level changes of long chain-polyunsaturated fatty acids in patients undergoing periodontal therapy combined with one year of omega-3 supplementation: a pilot randomized clinical trial. J Periodontal Implant Sci 2014;44:169-77.

13. Martuscelli G, Fiorellini JP, Crohin CC, Howell TH. The effect of interleukin-11 on the progression of ligature-induced periodontal disease in the beagle dog. J Periodontol 2000;71:573-8.

14. Offenbacher S, Odle BM, Van Dyke TE. The use of crevicular fluid prostaglandin E2 levels as a predictor of periodontal attachment loss. J Periodontal Res 1986;21:101-12.

15. Caton JG, Ciancio SG, Blieden TM, Bradshaw M, Crout RJ, Hefti AF, Massaro JM, Polson AM, Thomas J, Walker C. Treatment with subantimicrobial dose doxycycline improves the efficacy of scaling and root planing in patients with adult periodontitis. J Periodontol 2000;71:521-32.

16. Preshaw PM, Hefti AF, Novak MJ, Michalowicz BS, Pihlstrom BL, Schoor R, Trummel CL, Dean J, Van
Dyke TE, Walker CB, Bradshaw MH. Subantimicrobial dose doxycycline enhances the efficacy of scaling and root planing in chronic periodontitis: a multicenter trial. J Periodontol 2004;75:1068-76.

17. Reddy MS, Geurs NC, Gunsolley JC. Periodontal host modulation with antiproteinase, anti-inflammatory, and bone-sparing agents. A systematic review. Ann Periodontol 2003;8:12-37.

18. Higgins JP, Green S. Cochrane handbook for systematic reviews of interventions, vol. 5. Wiley Online Library, 2008.

19. Opengrey. System for information on grey literature in Europe. Vandoeuvre-lès-Nancy: Institut de l'Information Scientifique et Technique, 2011. Available from: http://www.opengrey.eu/ (updated 2015 Mar 11).

20. Armitage GC. Development of a classification system for periodontal diseases and conditions. Ann Periodontol 1999;4:1-6.

21. Cohen J. Weighted kappa: nominal scale agreement with provision for scaled disagreement or partial credit. Psychol Bull 1968;70:213-20.

22. Higgins JP, Altman DG, Gøtzsche PC, Jüni P, Moher D, Oxman AD, Savovic J, Schulz KF, Weeks L, Sterne JA; Cochrane Bias Methods Group; Cochrane Statistical Methods Group. The Cochrane Collaboration's tool for assessing risk of bias in randomised trials. BMJ 2011;343:d5928.

23. Clinicaltrials.gov. A service of the U.S. National Institutes of Health. Bethesda: U.S. National Library of Medicine, 2000. Available from: https://clinicaltrials.gov/ (updated 2015 Apr 5).

24. Higgins JP, Thompson SG, Deeks JJ, Altman DG. Measuring inconsistency in meta-analyses. BMJ 2003;327:557-60.

25. Aras H, Ca layan F, Güncü GN, Berbero lu A, Kilinç K. Effect of systemically administered naproxen sodium on clinical parameters and myeloperoxidase and elastase-like activity levels in gingival crevicular fluid. J Periodontol 2007;78:868-73.

26. Azoubel MC, Sarmento VA, Cangussú V, Azoubel E, Bittencourt S, Cunha FQ, Ribeiro RA, Brito GA. Adjunctive benefits of systemic etoricoxib in non-surgical treatment of aggressive periodontitis: short-term evaluation. J Periodontol 2008;79:1719- 
25.

27. Buduneli N, Buduneli E, Cetin EO, Kirilmaz L, Kütükçüler N. Clinical findings and gingival crevicular fluid prostaglandin E2 and interleukin-1-beta levels following initial periodontal treatment and short-term meloxicam administration. Expert Opin Pharmacother 2010;11:1805-12.

28. Jeffcoat MK, Page R, Reddy M, Wannawisute A, Waite P, Palcanis K, Cogen R, Williams RC, Basch C. Use of digital radiography to demonstrate the potential of naproxen as an adjunct in the treatment of rapidly progressive periodontitis. J Periodontal Res 1991;26:415-21.

29. Ng VW, Bissada NF. Clinical evaluation of systemic doxycycline and ibuprofen administration as an adjunctive treatment for adult periodontitis. J Periodontol 1998;69:772-6.

30. Ozgören O, Develioglu H, Güncü G, Akman A, Berker E. The adjunctive effect of tenoxicam during non-surgical periodontal treatment on clinical parameters and gingival crevicular fluid levels of MMP-8 and TNF-alpha in patients with chronic periodontitis - randomized, double-blind clinical trial. Adv Clin Exp Med 2014;23:559-65.

31. Reddy MS, Palcanis KG, Barnett ML, Haigh S, Charles $\mathrm{CH}$, Jeffcoat MK. Efficacy of meclofenamate sodium (Meclomen) in the treatment of rapidly progressive periodontitis. J Clin Periodontol 1993;20:635-40.

32. Taiyeb Ali TB, Waite IM. The effect of systemic ibuprofen on gingival inflammation in humans. J Clin Periodontol 1993;20:723-8.

33. Vardar S, Baylas H, Huseyinov A. Effects of selective cyclooxygenase-2 inhibition on gingival tissue levels of prostaglandin E2 and prostaglandin F2alpha and clinical parameters of chronic periodontitis. J Periodontol 2003;74:57-63.

34. Knowles JW, Burgett FG, Nissle RR, Shick RA, Morrison EC, Ramfjord SP. Results of periodontal treatment related to pocket depth and attachment level. Eight years. J Periodontol 1979;50:225-33.

35. Rostom A, Dube C, Wells G, Tugwell P, Welch V, Jolicoeur E, McGowan J. Prevention of NSAIDinduced gastroduodenal ulcers. Cochrane Database Syst Rev 2002;4:CD002296.

36. Slomiany BL, Slomiany A. Nonsteroidal antiinflammatory drugs impair oral mucosal repair by eliciting disturbances in endothelin-converting enzyme-1 and constitutive nitric oxide synthase. J Physiol Pharmacol 2001;52:81-92.

37. Kotsakis GA, Thai A, Ioannou AL, Demmer RT, Michalowicz BS. Association between low-dose aspirin and periodontal disease: results from the continuous national health and nutrition examination survey (NHANES) 2011-2012. J Clin Periodontol 2015;42:333-41.

38. Salvi GE, Lang NP. Host response modulation in the management of periodontal diseases. J Clin Periodontol 2005;32 Suppl 6:108-29.

39. Salvi GE, Lang NP. The effects of non-steroidal anti-inflammatory drugs (selective and non-selective) on the treatment of periodontal diseases. Curr Pharm Des 2005;11:1757-69.

40. Thomson WM, Williams SM. Partial- or full-mouth approaches to assessing the prevalence of and risk factors for periodontal disease in young adults. J Periodontol 2002;73:1010-4. 


\section{비스테로이드성 항염증제를 이용한 숙주조절이 치주질환에 미치는 영향: 체계적 고찰 및 메타 분석}

\section{강대영 ${ }^{1}$, 조인우 ${ }^{1}$, 신현승 ${ }^{1}$, 안형식 $^{2}$, 김현정 ${ }^{2 *}$, 박정철 ${ }^{1 *}$}

${ }^{1}$ 단국대학교 치과대학 치주과학교실

${ }^{2}$ 근거중심 의학연구소, 고려대학교 의과대학 예방의학교실

목적: 아라키돈산 대사물의 생합성을 억제하는 비스테로이드성 항염증제는 잠재적인 숙주조절제로 고려되고 있다. 이 종설의 목적은 비스테로이드성 항염증제를 비외과적 치주치료와 병용하였을 때 치주질환자에 미치는 영향을 평가하는 데 있다.

연구 재료 및 방법: 관련된 연구를 확인하기 위하여 세 전자 데이터베이스를 검색하였다. Cochrane의 고찰 방법론에 따 라 방법론적인 질, 임상 부착 수준과 탐침 깊이 변화량에 대한 평균 차이를 분석하였다.

결과: 총 12 개의 연구의 방법론을 평가하였고 이중 9 개의 연구에 대해 메타-분석을 시행하였다. 임상 부착 수준의 변화 에 대한 평균차의 경우 모든 관찰기간에서 비스테로이드성 항염증제 군과 대조군간 유의차를 보이지 않았다. 가장 큰 평 균차는 4주 때 $0.30 \mathrm{~mm}$ 로 나타났다 $(95 \%$ 신뢰구간 $=-0.37$ to 0.97$)$. 탐침 깊이 변화에 대한 평균차는 6 주 때 $0.34 \mathrm{~mm}$ ( $95 \%$ 신뢰구간 $=0.29$ to 0.40$)$ 로 유의차를 보였다.

결론: 이를 토대로 하였을 때 비스테로이드성 항염증제를 비외과적 치주치료와 병용하여 투여하였을 때 부가적으로 치 료효과를 증대시킬 수 있을 것으로 생각된다.

(구강회복응용과학지 2017;33(1):7-18)

주요어: 비스테로이드성 항염증제; 프로스타글란딘; 약물 요법; 치주 질환; 메타-분석

*교신저자: 김현정

(02841)서울시 성북구 인촌로 73 근거중심 의학연구소, 고려대학교 의과대학 예방의학교실

Tel: 02-2286-1341 | Fax: 02-2286-1342 | E-mail: moole@korea.ac. kr

*교신저자: 박정철

(31116)천안시 동남구 단대로 119 단국대학교 치과대학 치주과학교실

Tel: 041-550-0261 | Fax: 303-3442-7364 | E-mail: jcp@dent.dku. edu

접수일: 2017년 1월 6일 | 수정일: 2017년 3월 6일 | 채택일: 2017년 3월 9일 